\title{
Abstract
}

\section{Treatment of Severe Thrombocytopenia with Platelet Biotherapic and Phosphorus}

\author{
Bruna Scardoeli 1; Natália José Aith 2; Adalberto von Ancken 1; Cidéli de Paula Coelho 1,3** \\ 1 - High Dilution Science (HD Science), São Paulo, Brazil \\ 2 - United Metropolitan Colleges, São Paulo, Brazil \\ 3 - Santo Amaro University, São Paulo, Brazil \\ * bruna@esecon.com.br - ** https://orcid.org/0000-0002-0492-1822
}

Background Thrombocytopenia is a decrease in the number of platelets in the blood resulting from disorders of production, distribution or destruction, leading to bleeding. The respective treatment of thrombocytopenia is usually based on corticotherapy and platelet transfusion. Aims Report the evolution of homeopathic treatment in a 12 years old Maltese canine cardiopathic patient with thrombocytopenia associated with the diagnosis of Ehrlichia canis. Methods Replacement of corticoid and transfusion by Platelet Biotherapic, to stimulate platelet production and high diluted Phosphorus to contain the bleeding. Results On 01/28/19, the patient presented thrombocytopenia of $5.000 / \mathrm{mm}^{3}$ with large platelets, petechials and suffusion hemorrhages in dorsal and ventral regions, extensive acute cervical hematoma due to blood collection; anisocytosis, polychromasia and Howell Jolly corpuscle in red series; and also reactive lymphocytes, monocytes active and toxic neutrophils in white series. On 01/30/19, initiated the administration of 3 globules of Platelet Biotherapic 12CH every 12 hours; 3 globules of Phosphorus 6CH every 24 hours, both oral; and topical phytotherapeutic use of Arnica montana once daily. Three days later, it was observed the complete absence of petechials and suffusion hemorrhages, with elevation of platelets count to $74.000 / \mathrm{mm}^{3}$ and mild leukocytosis with improvement in overall patient well-being. On 02/06/19, there was regression of hematoma and leukocytosis, in addition to the platelet count in $95.000 / \mathrm{mm}^{3}$. On $02 / 14 / 19$, due to slight decrease in platelets to $92.000 / \mathrm{mm}^{3}$, the frequency of Phosphorus $6 \mathrm{CH}$ was changed every 12 hours. On 02/20/19, the new platelet count reached $126.000 / \mathrm{mm}^{3}$ with standardized morphology and no Howell Jolly record. Conclusion The homeopathies (High dilution medicine) have proven to be successful in the treatment of thrombocytopenia without causing side effects.

Keywords: Thrombocytopenia, Ehrlichia canis, Homeopathy, High Dilution

(C) International Journal of High Dilution Research.

Not for commercial purposes. 